\title{
A PARTICULAR BIDIMENSIONAL TIME-DEPENDENT RENEWAL RISK MODEL WITH CONSTANT INTEREST RATES
}

\author{
Ke-Ang Fu, Chang Ni and Hao Chen \\ School of Statistics and Mathematics, Zhejiang Gongshang University, Hangzhou 310018, China \\ E-mail: fukeang@hotmail.com
}

\begin{abstract}
Consider a particular bidimensional risk model, in which two insurance companies divide between them in different proportions both the premium income and the aggregate claims. In practice, it can be interpreted as an insurer-reinsurer scenario, where the reinsurer takes over a proportion of the insurer's losses. Under the assumption that the claim sizes and inter-arrival times form a sequence of independent and identically distributed random pairs, with each pair obeying a dependence structure, an asymptotic expression for the ruin probability of this bidimensional risk model with constant interest rates is established.
\end{abstract}

Keywords: bidimensional risk model, consistent variation, proportional reinsurance, ruin probability, time-dependence

\section{INTRODUCTION}

Consider a particular bidimensional risk model, in which two insurance companies split the amount they pay out of each claim in positive proportions $\delta_{1}$ and $\delta_{2}$ with $\delta_{1}+\delta_{2}=1$. The surplus of the $i$-th $(i=1,2)$ company up to time $t \geq 0$ is given by

$$
U_{i}(t)=x_{i} \mathrm{e}^{r_{i} t}+\int_{0}^{t} \mathrm{e}^{r_{i}(t-s)} \mathrm{d} C_{i}(s)-\delta_{i} \sum_{k=1}^{N(t)} X_{k} \mathrm{e}^{r_{i}\left(t-\tau_{k}\right)}, \quad i=1,2,
$$

where $\vec{r}=\left(r_{1}, r_{2}\right)$ is the vector of constant force of interest, $\vec{x}=\left(x_{1}, x_{2}\right)$ is the initial surplus vector, $\vec{C}(t)=\left(C_{1}(t), C_{2}(t)\right)$ is the vector of the total premium accumulated up to time $t$ with the nondecreasing and right continuous stochastic process satisfying $\vec{C}(0)=(0,0)$, and $\left\{X_{i} ; i \geq 1\right\}$ is the sequence of claim sizes whose common arrival times $\tau_{1}, \tau_{2}, \ldots$ constitute a renewal claim-number process $\{N(t) ; t \geq 0\}$ with finite renewal function $\lambda_{t}=\mathrm{E} N(t)=\sum_{i=1}^{\infty} \mathrm{P}\left(\tau_{i} \leq t\right)$. Note that this particular bidimensional risk model, firstly proposed by Avram et al. [2], where they focused on a special case that the loss process is a compound Poisson process with premiums only, is different from the usually-mentioned bidimensional risk model confronting a book of two dependent classes of business (see Chan et al. [5], Chen et al. [6-8], Gao and Yang[12], Jiang et al. [15], and references therein). It can be interpreted as an insurer-reinsurer scenario, where the reinsurer takes over a proportion of the insurer's losses. It is well-known that reinsurance contracts between a direct 
insurer and a reinsurer are used to transfer part of the risks assumed by the insurer, and proportional reinsurance is one of the main reinsurance forms. Under proportional reinsurance, the arrangement may be quota share reinsurance with a fixed percentage of each insurance policy being reinsured. Due to its wide applications in real life, this bidimensional risk model has been studied by many researchers, and one can refer to Badescu et al. [3], Foss et al. [10], Fu [11], Hu and Jiang [14], and Zhang and Wang [19] for more details.

Note that in reality if the deductible applied to each loss is raised, then the claim sizes would decrease and the inter-arrival time will increase, since small losses will be retained by the insured. Hence, it is reasonable to admit some kind of dependence structure between the claim sizes $\left\{X_{i} ; i \geq 1\right\}$ and their corresponding inter-arrival times $\left\{\theta_{i}=\tau_{i}-\tau_{i-1}\right.$; $i \geq 1\}\left(\tau_{0}=0\right)$, and in this paper, we use the dependence structure proposed by Asimit and Badescu [1], which is termed as time-dependence. For notational convenience, we state the assumption as follows.

Assumption A. The pairs of claim size and its corresponding inter-arrival time $\left(X_{i}, \theta_{i}\right), i \geq 1$, are independent and identically distributed (i.i.d.) copies of a generic pair $(X, \theta)$ with marginal distributions $F$ and $G$ on $[0, \infty)$, which fulfills the relation

$$
P(X>x \mid \theta=t) \sim P(X>x) h(t)
$$

uniformly for all $t \in[0, \infty)$ and for some measurable function $h(\cdot):[0, \infty) \rightarrow(0, \infty)$ with $\inf _{t \geq 0} h(t)>0$.

Note that to avoid triviality, both $F$ and $G$ are assumed not degenerate at 0 , and the symbol $\sim$ above means that the quotient of both sides tends to 1 as $x \rightarrow \infty$. When $t$ is not in the support of $\theta$, the conditional probability in (1.2) is understood as unconditional and $h(t)=1$ for such $t$. As discussed in Asimit and Badescu [1] and Li et al. [16], relation (1.2) defines a general dependence structure which includes many commonly-used bivariate copulas, for example, the Ali-Mikhail-Haq, Farlie-Gumbel-Morgenstern and Frank copulas, and allows for both negative and positive dependence.

As for this particular time-dependent bidimensional risk model, define the finite-time ruin probability as follows,

$$
\psi(\vec{x}, T)=\mathrm{P}\left(\tau\left(x_{1}, x_{2}\right) \leq T \mid U_{i}(0)=x_{i}, i=1,2\right),
$$

where $\tau\left(x_{1}, x_{2}\right)=\inf \left\{t: \max \left\{U_{1}(t), U_{2}(t)\right\}<0\right\}$ represents the time when $U_{1}(t)$ and $U_{2}(t)$ first become negative simultaneously; see Chan et al. [5].

In this paper, we aim to show the asymptotic behavior of (1.3) as $\vec{x} \rightarrow \infty$, i.e., $\left(x_{1}, x_{2}\right) \rightarrow$ $(\infty, \infty)$, with fixed $T$. In the rest of this paper, Section 2 presents our main result after introducing necessary preliminaries, and the proof of the main result with some crucial lemmas is exhibited in Section 3.

\section{NOTATION AND MAIN RESULTS}

In modeling extremal event, heavy-tailed risk has played an important role in insurance and finance, because it can describe large claims efficiently. We now introduce some related classes of heavy-tailed distributions. Denote the survival distribution of a random variable with distribution $F$ by $\bar{F}(x)=1-F(x)=\mathrm{P}(X>x)>0$. A useful heavy-tailed class is the class $\mathcal{L}$ of all distribution functions with long tail, characterized by the relation 
$\lim _{x \rightarrow \infty} \bar{F}(x+y) / \bar{F}(x)=1$ for any $y \in \mathbb{R}$. A subclass of $\mathcal{L}$ is the class $\mathcal{C}$ of consistently varying functions. By definition, $F \in \mathcal{C}$ if

$$
\lim _{y \searrow 1} \bar{F}_{*}(y)=1 \text { or, equivalently, } \lim _{y \nearrow 1} \bar{F}^{*}(y)=1,
$$

where $\bar{F}_{*}(y):=\liminf _{x \rightarrow \infty} \bar{F}(x y) / \bar{F}(x)$ and $\bar{F}^{*}(y):=\limsup _{x \rightarrow \infty} \bar{F}(x y) / \bar{F}(x)$. An important subclass of the class $\mathcal{C}$ is the class $\mathcal{R}_{-\alpha}$ of regularly varying functions specified by

$$
\lim _{x \rightarrow \infty} \frac{\bar{F}(x y)}{\bar{F}(x)}=y^{-\alpha}, \quad y \geq 1,
$$

for some $\alpha>0$. We use $\mathcal{R}$ to denote the union of all $\mathcal{R}_{-\alpha}$ over the range $0<\alpha<\infty$. It is noted that the class $\mathcal{R}$ contains lots of popular distributions such as Pareto, Burr, Loggamma, and $t$ distributions. In addition, $F$ on $[0, \infty)$ belongs to the dominated variation class by denoting $F \in \mathcal{D}$, if $\limsup _{x \rightarrow \infty} \bar{F}(x / 2) / \bar{F}(x)<\infty$. It is well known that the following inclusions are proper

$$
\mathcal{R} \subset \mathcal{C} \subset \mathcal{D} \cap \mathcal{L}
$$

see, Embrechts et al. [9] for more details.

Also we need two significant indices for any distribution $F$ supported on $[0, \infty)$. Set

$$
M^{*}(F)=-\lim _{y \rightarrow \infty} \frac{\log \bar{F}_{*}(y)}{\log y} \text { and } M_{*}(F)=-\lim _{y \rightarrow \infty} \frac{\log \bar{F}^{*}(y)}{\log y} .
$$

Following Tang and Tsitsiashvili [17], they are called the upper and lower Matuszewska indices, respectively. Generally, $0 \leq M_{*}(F) \leq M^{*}(F) \leq \infty$. If $F \in \mathcal{R}_{-\alpha}$, then $M^{*}(F)=$ $M_{*}(F)=\alpha$. Especially, if $F \in \mathcal{D}$, then $M^{*}(F)<\infty$.

Also note that it follows from (1.2) that $\mathrm{E} h(\theta)=1$. Hence, we define a random variable $\theta^{*}$ independent of $\left\{\left(X_{i}, \theta_{i}\right) ; i \geq 1\right\}$, with the distribution $G^{*}(\mathrm{~d} t)=h(t) G(\mathrm{~d} t)$. With interarrival times $\theta^{*}, \theta_{i}, i \geq 2$, we construct a delayed renewal counting process $\left\{N^{*}(t) ; t \geq 0\right\}$ with claim arrival time $\tau_{1}^{*}=\theta^{*}$ and $\tau_{k}^{*}=\theta^{*}+\sum_{i=2}^{k} \theta_{i}, \quad k=2,3, \ldots$ Denote by $\lambda_{t}^{*}$ the corresponding renewal function of $\left\{N^{*}(t) ; t \geq 0\right\}$, and it is easily seen that

$$
\lambda_{t}^{*}=\int_{0}^{t}\left(1+\lambda_{t-u}\right) h(u) G(\mathrm{~d} u)=\int_{0}^{t}\left(1+\lambda_{t-u}\right) G^{*}(\mathrm{~d} u) .
$$

Moreover, we assume that $\vec{C}(t)$ is independent of all source of randomness.

Hereafter, all limit relationships are for $\left(x_{1}, x_{2}\right) \rightarrow(\infty, \infty)(\vec{x} \rightarrow \infty)$ unless otherwise stated. For two positive functions $a\left(x_{1}, x_{2}\right)$ and $b\left(x_{1}, x_{2}\right)$, we write $a\left(x_{1}, x_{2}\right) \lesssim$ $b\left(x_{1}, x_{2}\right)$ or $b\left(x_{1}, x_{2}\right) \gtrsim a\left(x_{1}, x_{2}\right)$, if $\limsup a\left(x_{1}, x_{2}\right) / b\left(x_{1}, x_{2}\right) \leq 1, a\left(x_{1}, x_{2}\right) \sim b\left(x_{1}, x_{2}\right)$ if both $a\left(x_{1}, x_{2}\right) \lesssim b\left(x_{1}, x_{2}\right)$ and $a\left(x_{1}, x_{2}\right) \gtrsim b\left(x_{1}, x_{2}\right)$, and $a\left(x_{1}, x_{2}\right) \asymp b\left(x_{1}, x_{2}\right)$ if both $\lim \sup a\left(x_{1}, x_{2}\right) / b\left(x_{1}, x_{2}\right)<\infty$ and $\lim \sup b\left(x_{1}, x_{2}\right) / a\left(x_{1}, x_{2}\right)<\infty$.

Now we are ready to present our main result of this paper.

Theorem 2.1: Consider the bidimensional risk model given by (1.1) with Assumption A being fulfilled. If $F \in \mathcal{C}$ with $M_{*}(F)>0$, then for every $0<T<\infty$ satisfying $P\left(\tau_{1} \leq T\right)>0$, we have

$$
\psi(\vec{x}, T) \sim \int_{0}^{T} \bar{F}\left(\left(x_{1} \mathrm{e}^{r_{1} u} / \delta_{1}\right) \vee\left(x_{2} \mathrm{e}^{r_{2} u} / \delta_{2}\right)\right) \mathrm{d} \lambda_{u}^{*}
$$


Remark 2.1: From the proofs below, we can conclude that it allows to go beyond two insurance companies and consider a model with $d$-dimensional insurance contracts. In that case, under the conditions of Theorem 2.1, a similar proof of Theorem 2.1 will result in that the finite time ruin probability is asymptotically equivalent to $\int_{0}^{T} \bar{F}\left(\vee_{i=1}^{d}\left(x_{i} \mathrm{e}^{r_{i} u} / \delta_{i}\right)\right) \mathrm{d} \lambda_{u}^{*}$, where $x_{i}, r_{i}$ and $\delta_{i}(i=1, \ldots, d)$ are defined similarly to those in model (1.1).

In particular, if the two insurance companies invest all of their reserves into the same bond, then we have the following corollary immediately.

Corollary 2.1: Consider the bidimensional risk model given by (1.1) with Assumption A being fulfilled. Suppose that the insurance companies invest their reserves totally into a bond with constant force of interest $r>0$. If $F \in \mathcal{R}_{-\alpha}$ for some $\alpha>0$, then for every $0<T<\infty$ satisfying $P\left(\tau_{1} \leq T\right)>0$, we have

$$
\psi(\vec{x}, T) \sim \bar{F}\left(\frac{x_{1}}{\delta_{1}} \vee \frac{x_{2}}{\delta_{2}}\right) \int_{0}^{T} \mathrm{e}^{-r \alpha u} \mathrm{~d} \lambda_{u}^{*} .
$$

Remark 2.2: $\mathrm{Hu}$ and Jiang [14] and Zhang and Wang [19] have ever considered similar models with constant interest rates, and asymptotic expressions were derived under different conditions. Theorem 2.1 and Corollary 2.1 allow for the dependence between the claim sizes and their corresponding inter-arrival times, which extend the results of $\mathrm{Hu}$ and Jiang [14] and Zhang and Wang [19] to a more general and practical setting. From Remark 2.1, it follows, under the conditions of Corollary 2.1, the finite time ruin probability of a similar model with $d$-dimensional insurance contracts is asymptotically equivalent to $\bar{F}\left(\vee_{i=1}^{d} x_{i} / \delta_{i}\right) \int_{0}^{T} \mathrm{e}^{-r \alpha u} \mathrm{~d} \lambda_{u}^{*}$.

\section{PROOFS}

If $F \in \mathcal{D}$, then from Proposition 2.2.1 in Bingham et al. [4] that, for any $p_{1}<M_{*}(F)$ and $p_{2}>M^{*}(F)$, there are positive constants $c_{i}$ and $d, i=1,2$, such that the two-side inequality

$$
c_{1}(x / y)^{p_{1}} \leq \bar{F}(y) / \bar{F}(x) \leq c_{2}(x / y)^{p_{2}}
$$

holds for all $x \geq y \geq d$. Furthermore, fixing the variable $y$ in (3.1) gives $x^{-p}=o(\bar{F}(x))$ for $p>M^{*}(F)$. In what follows, $\bar{A}(x) \asymp \bar{B}(x)$ means both $\lim \sup _{x \rightarrow \infty} \bar{A}(x) / \bar{B}(x)<\infty$ and $\lim \sup _{x \rightarrow \infty} \bar{B}(x) / \bar{A}(x)<\infty$.

For proving the main result, we need the following series of lemmas. Besides their critical role in the proof of Theorem 2.1, some of these lemmas are themselves interesting. Among them, Lemma 3.1 is from Lemma 5.7 of Tang and Yuan [18], which will be used frequently in the proofs.

Lemma 3.1: Let $X$ be a random variable with a dominatedly-varying right tail and upper Matuszewska index $M^{*}(F)$ ( $F$ is the distribution function of $X$ ), let $\theta$ be a nonnegative random variable with $E \theta^{\beta}<\infty$ for some $\beta>M^{*}(F)$, let $\left\{\Delta_{t}, t \in \mathcal{T}\right\}$ be a set of random events satisfying $\lim _{t \rightarrow t_{0}} P\left(\Delta_{t}\right)=0$ for some $t_{0}$ in the closure of the index set $\mathcal{T}$, and let $\left\{\theta,\left\{\Delta_{t}, t \in \mathcal{T}\right\}\right\}$ be independent of $X$. Then

$$
\lim _{t \rightarrow t_{0}} \limsup _{x \rightarrow \infty} \frac{P\left(\theta X>x, \Delta_{t}\right)}{P(\theta X>x)}=\lim _{t \rightarrow t_{0}} \limsup _{x \rightarrow \infty} \frac{P\left(\theta X>x, \Delta_{t}\right)}{P(X>x)}=0 .
$$


Lemma 3.2: Under the conditions of Theorem 2.1, it holds, for any fixed $n$ and $0<T<\infty$,

$$
\begin{array}{r}
P\left(\sum_{i=1}^{n} X_{i} \mathrm{e}^{-r_{1} \tau_{i}}>x_{1}, \sum_{j=1}^{n} X_{j} \mathrm{e}^{-r_{2} \tau_{j}}>x_{2}, N(T)=n\right) \\
\quad \sim \sum_{i=1}^{n} P\left(X_{i} \mathrm{e}^{-r_{1} \tau_{i}}>x_{1}, X_{i} \mathrm{e}^{-r_{2} \tau_{i}}>x_{2}, N(T)=n\right) .
\end{array}
$$

Proof: Note that the event $\{N(T)=n\}$ in relation (3.2) could have a probability 0 . In the proof below, we still use the notation $a\left(x_{1}, x_{2}, T\right) \sim b\left(x_{1}, x_{2}, T\right)$ even though the two functions $a\left(x_{1}, x_{2}, T\right)$ and $b\left(x_{1}, x_{2}, T\right)$ could be simultaneously equal to 0 . For such an occasion the notation exactly means $a\left(x_{1}, x_{2}, T\right)=(1+o(1)) b\left(x_{1}, x_{2}, T\right)$.

For any $0<\varepsilon<1$, we have

$$
\begin{aligned}
& \mathrm{P}\left(\sum_{i=1}^{n} X_{i} \mathrm{e}^{-r_{1} \tau_{i}}>x_{1}, \sum_{j=1}^{n} X_{j} \mathrm{e}^{-r_{2} \tau_{j}}>x_{2}, N(T)=n\right) \\
& \leq \sum_{i=1}^{n} \sum_{j=1}^{n} \mathrm{P}\left(X_{i} \mathrm{e}^{-r_{1} \tau_{i}}>(1-\varepsilon) x_{1}, X_{j} \mathrm{e}^{-r_{2} \tau_{j}}>(1-\varepsilon) x_{2}, N(T)=n\right) \\
& \quad+\mathrm{P}\left(\sum_{i=1}^{n} X_{i} \mathrm{e}^{-r_{1} \tau_{i}}>x_{1}, \sum_{j=1}^{n} X_{j} \mathrm{e}^{-r_{2} \tau_{j}}>x_{2}, \bigcap_{j=1}^{n}\left(X_{j} \mathrm{e}^{-r_{2} \tau_{j}} \leq(1-\varepsilon) x_{2}\right), N(T)=n\right) \\
& \quad+\mathrm{P}\left(\sum_{i=1}^{n} X_{i} \mathrm{e}^{-r_{1} \tau_{i}}>x_{1}, \sum_{j=1}^{n} X_{j} \mathrm{e}^{-r_{2} \tau_{j}}>x_{2}, \bigcap_{i=1}^{n}\left(X_{i} \mathrm{e}^{-r_{1} \tau_{i}} \leq(1-\varepsilon) x_{1}\right), N(T)=n\right) \\
& =:
\end{aligned}
$$

As for $I_{1}$, we have

$$
\begin{aligned}
I_{1} \leq & \sum_{i=1}^{n} \mathrm{P}\left(X_{i} \mathrm{e}^{-r_{1} \tau_{i}}>(1-\varepsilon) x_{1}, X_{i} \mathrm{e}^{-r_{2} \tau_{i}}>(1-\varepsilon) x_{2}, N(T)=n\right) \\
& +\sum_{1 \leq i \neq j \leq n} \mathrm{P}\left(X_{i}>x_{1}, X_{j}>x_{2}, N(T)=n\right) \\
= & \sum_{i=1}^{n} P_{i i}+\sum_{1 \leq i \neq j \leq n} P_{i j} .
\end{aligned}
$$

Note that

$$
P_{i i} \geq \mathrm{P}\left(X_{i} \mathrm{e}^{-r_{1} T}>(1-\varepsilon)\left(x_{1} \vee x_{2}\right), N(T)=n\right) \asymp \mathrm{P}\left(X_{i}>x_{1} \vee x_{2}\right),
$$

and $P_{i j}=o\left(\mathrm{P}\left(X_{i}>x_{1}\right)\right), P_{i j}=o\left(\mathrm{P}\left(X_{j}>x_{2}\right)\right)$, deduced from Lemma 3.1. This implies $P_{i j} / P_{i i} \rightarrow 0$ as $\vec{x} \rightarrow \infty$, which, coupled with

$$
\begin{aligned}
P_{i i}= & \int_{\Omega_{n}(T)} \ldots \int \mathrm{P}\left(X_{i} \mathrm{e}^{-r_{1} t_{i}}>(1-\varepsilon) x_{1}, X_{i} \mathrm{e}^{-r_{2} t_{i}}>(1-\varepsilon) x_{2} \mid \theta_{i}=s_{i}\right) \\
& \times \bar{G}\left(T-t_{n}\right) \prod_{k=1}^{n} G\left(\mathrm{~d} s_{k}\right)
\end{aligned}
$$




$$
\begin{aligned}
& \sim \int_{\Omega_{n}(T)} \ldots \int \mathrm{P}\left(X_{i} \mathrm{e}^{-r_{1} t_{i}}>(1-\varepsilon) x_{1}, X_{i} \mathrm{e}^{-r_{2} t_{i}}>(1-\varepsilon) x_{2}\right) h\left(s_{i}\right) \bar{G}\left(T-t_{n}\right) \prod_{k=1}^{n} G\left(\mathrm{~d} s_{k}\right) \\
& \lesssim \frac{1}{\bar{F}_{*}\left((1-\varepsilon)^{-1}\right)} \int_{\Omega_{n}(T)} \ldots \int \mathrm{P}\left(X_{i} \mathrm{e}^{-r_{1} t_{i}}>x_{1}, X_{i} \mathrm{e}^{-r_{2} t_{i}}>x_{2}\right) h\left(s_{i}\right) \bar{G}\left(T-t_{n}\right) \prod_{k=1}^{n} G\left(\mathrm{~d} s_{k}\right) \\
& =\frac{1}{\bar{F}_{*}\left((1-\varepsilon)^{-1}\right)} \mathrm{P}\left(X_{i} \mathrm{e}^{-r_{1} \tau_{i}}>x_{1}, X_{i} \mathrm{e}^{-r_{2} \tau_{i}}>x_{2}, N(T)=n\right),
\end{aligned}
$$

where $\Omega_{n}(t)=\left\{\left(s_{1}, \ldots, s_{n}\right) \in[0, t]^{n}: t_{n}=\sum_{k=1}^{n} s_{k} \leq t\right\}$ and Assumption $\mathrm{A}$ is applied, gives

$$
I_{1} \lesssim \frac{1}{\bar{F}_{*}\left((1-\varepsilon)^{-1}\right)} \sum_{i=1}^{n} \mathrm{P}\left(X_{i} \mathrm{e}^{-r_{1} \tau_{i}}>x_{1}, X_{i} \mathrm{e}^{-r_{2} \tau_{i}}>x_{2}, N(T)=n\right)
$$

While for $I_{2}$, we have

$$
\begin{aligned}
I_{2} \leq & \sum_{i=1}^{n} \sum_{j=1}^{n} \sum_{\substack{1 \leq k \leq n \\
k \neq j}} \\
& \times \mathrm{P}\left(X_{i} \mathrm{e}^{-r_{1} \tau_{i}}>\frac{x_{1}}{n}, \frac{x_{2}}{n}<X_{j} \mathrm{e}^{-r_{2} \tau_{j}}<(1-\varepsilon) x_{2}, X_{k} \mathrm{e}^{-r_{2} \tau_{k}}>\frac{\varepsilon x_{2}}{n-1}, N(T)=n\right) \\
\leq & \sum_{i=1}^{n} \sum_{\substack{1 \leq k \leq n \\
k \neq i}} \mathrm{P}\left(X_{i} \mathrm{e}^{-r_{1} \tau_{i}}>\frac{x_{1}}{n}, X_{i} \mathrm{e}^{-r_{2} \tau_{i}}>\frac{x_{2}}{n}, X_{k} \mathrm{e}^{-r_{2} \tau_{k}}>\frac{\varepsilon x_{2}}{n-1}, N(T)=n\right) \\
& +\sum_{i=1}^{n} \sum_{\substack{1 \leq j \leq n \\
j \neq i}} \sum_{\substack{1 \leq k \leq n \\
k \neq j}} \mathrm{P}\left(X_{i} \mathrm{e}^{-r_{1} \tau_{i}}>\frac{x_{1}}{n}, X_{k} \mathrm{e}^{-r_{2} \tau_{k}}>\frac{\varepsilon x_{2}}{n-1}, X_{j} \mathrm{e}^{-r_{2} \tau_{j}}>\frac{x_{2}}{n}, N(T)=n\right) \\
= & o\left(\sum_{i=1}^{n} P_{i i}\right)=o\left(I_{1}\right),
\end{aligned}
$$

where in the last step Lemma 3.1 with (3.3) is applied. Taking a similar proof, we can obtain $I_{3}=o\left(I_{1}\right)$. Hence, by letting first $\vec{x} \rightarrow \infty$ and then $\varepsilon \rightarrow 0$, with $F \in \mathcal{C}$, we can get

$$
\begin{gathered}
\mathrm{P}\left(\sum_{i=1}^{n} X_{i} \mathrm{e}^{-r_{1} \tau_{i}}>x_{1}, \sum_{j=1}^{n} X_{j} \mathrm{e}^{-r_{2} \tau_{j}}>x_{2}, N(T)=n\right) \\
\quad \lesssim \sum_{i=1}^{n} \mathrm{P}\left(X_{i} \mathrm{e}^{-r_{1} \tau_{i}}>x_{1}, X_{i} \mathrm{e}^{-r_{2} \tau_{i}}>x_{2}, N(T)=n\right) .
\end{gathered}
$$


As for the asymptotic lower bound, we have, for any $0<\varepsilon<1$,

$$
\begin{aligned}
& \mathrm{P}\left(\sum_{i=1}^{n} X_{i} \mathrm{e}^{-r_{1} \tau_{i}}>x_{1}, \sum_{j=1}^{n} X_{j} \mathrm{e}^{-r_{2} \tau_{j}}>x_{2}, N(T)=n\right) \\
& \geq \mathrm{P}\left(\sum_{i=1}^{n} X_{i} \mathrm{e}^{-r_{1} \tau_{i}}>x_{1}, \sum_{j=1}^{n} X_{j} \mathrm{e}^{-r_{2} \tau_{j}}>x_{2}, \max _{1 \leq i \leq n} X_{i} \mathrm{e}^{-r_{1} \tau_{i}}>(1+\varepsilon) x_{1},\right. \\
& \left.\max _{1 \leq j \leq n} X_{j} \mathrm{e}^{-r_{2} \tau_{j}}>(1+\varepsilon) x_{2}, N(T)=n\right) \\
& \geq \sum_{i=1}^{n} \mathrm{P}\left(X_{i} \mathrm{e}^{-r_{1} \tau_{i}}>(1+\varepsilon) x_{1}, X_{i} \mathrm{e}^{-r_{2} \tau_{i}}>(1+\varepsilon) x_{2}, N(T)=n\right) \\
& +\sum_{1 \leq i \neq j \leq n} \mathrm{P}\left(X_{i} \mathrm{e}^{-r_{1} T}>(1+\varepsilon) x_{1}, X_{j} \mathrm{e}^{-r_{2} T}>(1+\varepsilon) x_{2}, N(T)=n\right) \\
& -\sum_{i=1}^{n} \sum_{j=1}^{n} \sum_{\substack{1 \leq k \leq n \\
k \neq j}} \mathrm{P}\left(X_{i} \mathrm{e}^{-r_{1} \tau_{i}}>(1+\varepsilon) x_{1}, X_{j} \mathrm{e}^{-r_{2} \tau_{j}}>(1+\varepsilon) x_{2},\right. \\
& \left.X_{k} \mathrm{e}^{-r_{2} \tau_{k}}>(1+\varepsilon) x_{2}, N(T)=n\right) \\
& -\sum_{k=1}^{n} \sum_{j=1}^{n} \sum_{\substack{1 \leq i \leq n \\
i \neq j}} \mathrm{P}\left(X_{i} \mathrm{e}^{-r_{1} \tau_{i}}>(1+\varepsilon) x_{1}, X_{j} \mathrm{e}^{-r_{1} \tau_{j}}>(1+\varepsilon) x_{1},\right. \\
& \left.X_{k} \mathrm{e}^{-r_{2} \tau_{k}}>(1+\varepsilon) x_{2}, N(T)=n\right) \\
& =(1+o(1)) \sum_{i=1}^{n} \mathrm{P}\left(X_{i} \mathrm{e}^{-r_{1} \tau_{i}}>(1+\varepsilon) x_{1}, X_{i} \mathrm{e}^{-r_{2} \tau_{i}}>(1+\varepsilon) x_{2}, N(T)=n\right),
\end{aligned}
$$

where in the last step Lemma 3.1 is applied again. By taking a similar way to the proof of (3.4), we can get

$$
\begin{aligned}
& \mathrm{P}\left(\sum_{i=1}^{n} X_{i} \mathrm{e}^{-r_{1} \tau_{i}}>x_{1}, \sum_{j=1}^{n} X_{j} \mathrm{e}^{-r_{2} \tau_{j}}>x_{2}, N(T)=n\right) \\
& \quad \gtrsim \bar{F}_{*}(1+\varepsilon) \sum_{i=1}^{n} \mathrm{P}\left(X_{i} \mathrm{e}^{-r_{1} \tau_{i}}>x_{1}, X_{i} \mathrm{e}^{-r_{2} \tau_{i}}>x_{2}, N(T)=n\right) .
\end{aligned}
$$

Hence, by letting first $\vec{x} \rightarrow \infty$ and then $\varepsilon \rightarrow 0$ with $F \in \mathcal{C}$, we have

$$
\begin{gathered}
\mathrm{P}\left(\sum_{i=1}^{n} X_{i} \mathrm{e}^{-r_{1} \tau_{i}}>x_{1}, \sum_{j=1}^{n} X_{j} \mathrm{e}^{-r_{2} \tau_{j}}>x_{2}, N(T)=n\right) \\
\gtrsim \sum_{i=1}^{n} \mathrm{P}\left(X_{i} \mathrm{e}^{-r_{1} \tau_{i}}>x_{1}, X_{i} \mathrm{e}^{-r_{2} \tau_{i}}>x_{2}, N(T)=n\right) .
\end{gathered}
$$

This, together with (3.5), implies (3.2) holds, and the proof of Lemma 3.2 is finished. 
Lemma 3.3: Let the pairs of claim size and its corresponding inter-arrival time $\left(X_{i}, \theta_{i}\right), i \geq$ 1 , be i.i.d. random vectors and Assumption $A$ is fulfilled. Then under the conditions of Theorem 2.1, it holds, for every $0<T<\infty$ satisfying $P\left(\tau_{1} \leq T\right)>0$,

$$
\begin{gathered}
P\left(\sum_{i=1}^{N(T)} X_{i} \mathrm{e}^{-r_{1} \tau_{i}}>x_{1} / \delta_{1}, \sum_{j=1}^{N(T)} X_{j} \mathrm{e}^{-r_{2} \tau_{j}}>x_{2} / \delta_{2}\right) \\
\sim \int_{0}^{T} \bar{F}\left(\left(x_{1} \mathrm{e}^{r_{1} u} / \delta_{1}\right) \vee\left(x_{2} \mathrm{e}^{r_{2} u} / \delta_{2}\right)\right) \mathrm{d} \lambda_{u}^{*}
\end{gathered}
$$

Proof: Arbitrarily choose some positive integer $M$. Note that

$$
\begin{aligned}
& \mathrm{P}\left(\sum_{i=1}^{N(T)} X_{i} \mathrm{e}^{-r_{1} \tau_{i}}>x_{1} / \delta_{1}, \sum_{j=1}^{N(T)} X_{j} \mathrm{e}^{-r_{2} \tau_{j}}>x_{2} / \delta_{2}\right) \\
& =\left(\sum_{n=1}^{M}+\sum_{n=M+1}^{\infty}\right) \mathrm{P}\left(\sum_{i=1}^{n} X_{i} \mathrm{e}^{-r_{1} \tau_{i}}>x_{1} / \delta_{1}, \sum_{j=1}^{n} X_{j} \mathrm{e}^{-r_{2} \tau_{j}}>x_{2} / \delta_{2}, N(T)=n\right) \\
& =: I_{4}+I_{5} .
\end{aligned}
$$

First consider $I_{4}$. By applying Lemma 3.2, we arrive at

$$
\begin{aligned}
I_{4} & \sim \sum_{n=1}^{M} \sum_{i=1}^{n} \mathrm{P}\left(X_{i} \mathrm{e}^{-r_{1} \tau_{i}}>x_{1} / \delta_{1}, X_{i} \mathrm{e}^{-r_{2} \tau_{i}}>x_{2} / \delta_{2}, N(T)=n\right) \\
& =\left(\sum_{n=1}^{\infty}-\sum_{n=M+1}^{\infty}\right) \sum_{i=1}^{n} \mathrm{P}\left(X_{i} \mathrm{e}^{-r_{1} \tau_{i}}>x_{1} / \delta_{1}, X_{i} \mathrm{e}^{-r_{2} \tau_{i}}>x_{2} / \delta_{2}, N(T)=n\right) \\
& =: I_{41}-I_{42},
\end{aligned}
$$

Let $\left\{\theta_{i}^{*} ; i \geq 1\right\}$ be the i.i.d. copies of $\theta^{*}$, independent of $\left\{\left(X_{i}, \theta_{i}\right) ; i \geq 1\right\}$. Then by interchanging the order of the sums, we can get that

$$
\begin{aligned}
I_{41}= & \sum_{i=1}^{\infty} \int_{0}^{T} \int_{0}^{T-u} \mathrm{P}\left(X_{i} \mathrm{e}^{-r_{1}(u+v)}>x_{1} / \delta_{1}, X_{i} \mathrm{e}^{-r_{2}(u+v)}>x_{2} / \delta_{2} \mid \theta_{i}=u\right) \\
& \times \mathrm{P}\left(\tau_{i-1} \in \mathrm{d} v\right) \mathrm{P}\left(\theta_{i} \in \mathrm{d} u\right) \\
\sim & \sum_{i=1}^{\infty} \int_{0}^{T} \int_{0}^{T-u} \mathrm{P}\left(X_{i} \mathrm{e}^{-r_{1}(u+v)}>x_{1} / \delta_{1}, X_{i} \mathrm{e}^{-r_{2}(u+v)}>x_{2} / \delta_{2}\right) \\
& \times \mathrm{P}\left(\tau_{i-1} \in \mathrm{d} v\right) h(u) \mathrm{P}\left(\theta_{i} \in \mathrm{d} u\right) \\
= & \sum_{i=1}^{\infty} \int_{0}^{T} \int_{0}^{T-u} \mathrm{P}\left(X_{i} \mathrm{e}^{-r_{1}(u+v)}>x_{1} / \delta_{1}, X_{i} \mathrm{e}^{-r_{2}(u+v)}>x_{2} / \delta_{2}\right) \mathrm{P}\left(\tau_{i-1} \in \mathrm{d} v\right) \mathrm{P}\left(\theta_{i}^{*} \in \mathrm{d} u\right)
\end{aligned}
$$




$$
\begin{aligned}
& =\sum_{i=1}^{\infty} \mathrm{P}\left(X_{i} \mathrm{e}^{-r_{1}\left(\tau_{i-1}+\theta_{i}^{*}\right)}>x_{1} / \delta_{1}, X_{i} \mathrm{e}^{-r_{2}\left(\tau_{i-1}+\theta_{i}^{*}\right)}>x_{2} / \delta_{2}, \tau_{i-1}+\theta_{i}^{*} \leq T\right) \\
& =\int_{0}^{T} \bar{F}\left(\left(x_{1} \mathrm{e}^{r_{1} u} / \delta_{1}\right) \vee\left(x_{2} \mathrm{e}^{r_{2} u} / \delta_{2}\right)\right) \sum_{i=1}^{\infty} \mathrm{P}\left(\tau_{i}^{*} \in \mathrm{d} u\right) \\
& =\int_{0}^{T} \bar{F}\left(\left(x_{1} \mathrm{e}^{r_{1} u} / \delta_{1}\right) \vee\left(x_{2} \mathrm{e}^{r_{2} u} / \delta_{2}\right)\right) \mathrm{d} \lambda_{u}^{*},
\end{aligned}
$$

since $\tau_{i-1}+\theta_{i}^{*} \stackrel{d}{=} \tau_{i}^{*}$ with $\stackrel{d}{=}$ denoting equality in distribution, where Assumption A is used in the second step.

As for $I_{42}$, we have

$$
\begin{aligned}
I_{42} & \leq \sum_{n=M+1}^{\infty} \sum_{i=1}^{n} \mathrm{P}\left(X_{i}>\frac{x_{1}}{\delta_{1}} \vee \frac{x_{2}}{\delta_{2}}, \tau_{n}-\theta_{i} \leq T\right) \\
& \leq \sum_{n=M+1}^{\infty} n \bar{F}\left(\frac{x_{1}}{\delta_{1}} \vee \frac{x_{2}}{\delta_{2}}\right) \mathrm{P}\left(\tau_{n-1} \leq T\right) \\
& \leq \sum_{n=M+1}^{\infty} n \bar{F}\left(\frac{x_{1}}{\delta_{1}} \vee \frac{x_{2}}{\delta_{2}}\right) \mathrm{P}(N(T) \geq n-1) \\
& \leq \bar{F}\left(\frac{x_{1}}{\delta_{1}} \vee \frac{x_{2}}{\delta_{2}}\right) \mathrm{E}(N(T)+1)^{2} I(N(T) \geq M),
\end{aligned}
$$

where $I(\cdot)$ is an indicator function. This leads to $\lim _{M \rightarrow \infty} \lim _{\vec{x} \rightarrow \infty} I_{42} / I_{41}=0$, where $\mathrm{E}(N(T))^{Q}<\infty$ for any $Q>0$, deduced from Lemma 4.2 of Hao and Tang [13], is employed. Hence, to complete the proof, it is sufficient to show $I_{5}=o\left(I_{4}\right)$, as first $\vec{x} \rightarrow \infty$ and then $M \rightarrow \infty$.

Now we begin to deal with $I_{5}$. Find an $N$ such that $\sum_{i=1}^{\infty} 1 / i^{2}<N$, and then it follows that, for $x_{1}, x_{2}$ large enough and $p_{2}>M^{*}(F)$,

$$
\begin{aligned}
& I_{5} \leq \sum_{n=M+1}^{\infty} \mathrm{P}\left(\sum_{i=1}^{n} X_{i} \mathrm{e}^{-r_{1} \tau_{i}}>\sum_{i=1}^{n} \frac{x_{1}}{i^{2} N \delta_{1}}, \sum_{j=1}^{n} X_{j} \mathrm{e}^{-r_{2} \tau_{j}}>\sum_{j=1}^{n} \frac{x_{2}}{j^{2} N \delta_{2}}, \tau_{n} \leq T\right) \\
& \leq \sum_{n=M+1}^{\infty} \sum_{i=1}^{n} \sum_{j=1}^{n} \mathrm{P}\left(X_{i}>\frac{x_{1}}{i^{2} N \delta_{1}}, X_{j}>\frac{x_{2}}{j^{2} N \delta_{2}}, \tau_{n} \leq T\right) \\
& \leq \sum_{n=M+1}^{\infty}\left(\sum_{i=1}^{n} \bar{F}\left(\frac{1}{i^{2} N}\left(\frac{x_{1}}{\delta_{1}} \vee \frac{x_{2}}{\delta_{2}}\right)\right) \mathrm{P}\left(\tau_{n-1} \leq T\right)\right. \\
& \left.+\sum_{1 \leq i \neq j \leq n} \bar{F}\left(\frac{x_{1}}{i^{2} N \delta_{1}}\right) \bar{F}\left(\frac{x_{2}}{j^{2} N \delta_{2}}\right) \mathrm{P}\left(\tau_{n-2} \leq T\right)\right) \\
& \leq C \sum_{n=M+1}^{\infty}\left(\sum_{i=1}^{n}\left(i^{2} N\right)^{p_{2}} \bar{F}\left(\frac{x_{1}}{\delta_{1}} \vee \frac{x_{2}}{\delta_{2}}\right) \mathrm{P}(N(T) \geq n-1)\right. \\
& \left.+\sum_{1 \leq i \neq j \leq n}(i j N)^{2 p_{2}} \bar{F}\left(x_{1}\right) \bar{F}\left(x_{2}\right) \mathrm{P}(N(T) \geq n-2)\right)
\end{aligned}
$$




$$
\begin{aligned}
\leq & C\left(\bar{F}\left(\frac{x_{1}}{\delta_{1}} \vee \frac{x_{2}}{\delta_{2}}\right) \mathrm{E}(N(T)+1)^{2 p_{2}+1} I(N(T) \geq M)\right. \\
& \left.+\bar{F}\left(x_{1}\right) \bar{F}\left(x_{2}\right) \mathrm{E}(N(T)+2)^{4 p_{2}+3} I(N(T) \geq M-1)\right),
\end{aligned}
$$

where (3.1) is used in the fourth step, and $C$ denotes a generic constant that may be different in each of its appearance. Applying $\mathrm{E}(N(T))^{Q}<\infty$ for any $Q>0$ again, it results in $\lim _{M \rightarrow \infty} \lim _{\vec{x} \rightarrow \infty} I_{5} / I_{41}=0$, which implies $I_{5}=o\left(I_{4}\right)$, as first $\vec{x} \rightarrow \infty$ and then $M \rightarrow \infty$, and the proof of Lemma 3.3 is completed.

Now we begin to prove Theorem 2.1.

Proof of Theorem 2.1: Observe that

$$
\begin{aligned}
\psi(\vec{x}, T) & =\mathrm{P}\left(U_{1}(t)<0, U_{2}(t)<0 \quad \text { for some } 0 \leq t \leq T \mid U_{i}(0)=x_{i}, i=1,2\right) \\
& =\mathrm{P}\left(\inf _{0 \leq t \leq T} \max \left(x_{i}+\int_{0}^{t} \mathrm{e}^{-r_{i} s} \mathrm{~d} C_{i}(s)-\delta_{i} \sum_{k=1}^{N(t)} X_{k} \mathrm{e}^{-r_{i} \tau_{k}}, i=1,2\right)<0\right) .
\end{aligned}
$$

Then by applying Lemma 3.3, it gives

$$
\begin{aligned}
\psi(\vec{x}, T) & \leq \mathrm{P}\left(\sum_{i=1}^{N(T)} X_{i} \mathrm{e}^{-r_{1} \tau_{i}}>x_{1} / \delta_{1}, \sum_{j=1}^{N(T)} X_{j} \mathrm{e}^{-r_{2} \tau_{j}}>x_{2} / \delta_{2}\right) \\
& \sim \int_{0}^{T} \bar{F}\left(\left(x_{1} \mathrm{e}^{r_{1} u} / \delta_{1}\right) \vee\left(x_{2} \mathrm{e}^{r_{2} u} / \delta_{2}\right)\right) \mathrm{d} \lambda_{u}^{*} .
\end{aligned}
$$

Denote by $H(\cdot, \cdot)$ the joint distribution function of $\left(\int_{0}^{\infty} \mathrm{e}^{-r_{1} s} C_{1}(\mathrm{~d} s), \int_{0}^{\infty} \mathrm{e}^{-r_{2} s} C_{2}(\mathrm{~d} s)\right)$, and then an application of Lemma 3.3 leads to

$$
\begin{aligned}
\psi(\vec{x}, T) & \geq \mathrm{P}\left(\sum_{k=1}^{N(T)} X_{k} \mathrm{e}^{-r_{i} \tau_{k}}>\left(x_{i}+\int_{0}^{\infty} \mathrm{e}^{-r_{i} s} C_{i}(\mathrm{~d} s)\right) / \delta_{i}, i=1,2\right) \\
& =\iint_{0<s_{1}, s_{2}<\infty} \mathrm{P}\left(\sum_{k=1}^{N(T)} X_{k} \mathrm{e}^{-r_{i} \tau_{k}}>\left(x_{i}+s_{i}\right) / \delta_{i}, i=1,2\right) H\left(\mathrm{~d} s_{1}, \mathrm{~d} s_{2}\right) \\
& \sim \iint_{0<s_{1}, s_{2}<\infty} \int_{0}^{T} \bar{F}\left(\left(\left(x_{1}+s_{1}\right) \mathrm{e}^{r_{1} u} / \delta_{1}\right) \vee\left(\left(x_{2}+s_{2}\right) \mathrm{e}^{r_{2} u} / \delta_{2}\right)\right) \mathrm{d} \lambda_{u}^{*} H\left(\mathrm{~d} s_{1}, \mathrm{~d} s_{2}\right) .
\end{aligned}
$$

By making use of $F \in \mathcal{C} \subset \mathcal{L}$, we have

$$
\int_{0}^{T} \bar{F}\left(\left(\left(x_{1}+s_{1}\right) \mathrm{e}^{r_{1} u} / \delta_{1}\right) \vee\left(\left(x_{2}+s_{2}\right) \mathrm{e}^{r_{2} u} / \delta_{2}\right)\right) \mathrm{d} \lambda_{u}^{*} \sim \int_{0}^{T} \bar{F}\left(\left(x_{1} \mathrm{e}^{r_{1} u} / \delta_{1}\right) \vee\left(x_{2} \mathrm{e}^{r_{2} u} / \delta_{2}\right)\right) \mathrm{d} \lambda_{u}^{*},
$$

which, coupled with the dominated convergence theorem, guarantees

$$
\psi(\vec{x}, T) \gtrsim \int_{0}^{T} \bar{F}\left(\left(x_{1} \mathrm{e}^{r_{1} u} / \delta_{1}\right) \vee\left(x_{2} \mathrm{e}^{r_{2} u} / \delta_{2}\right)\right) \mathrm{d} \lambda_{u}^{*} .
$$

This, combined with (3.9), completes the proof of Theorem 2.1. 


\section{Acknowledgments}

The authors thank the referees for pointing out some errors in a previous version, as well as for several comments that have led to improvements in this work. Project supported by the Humanities and Social Sciences Foundation of the Ministry of Education of China (No. 17YJC910002) and First Class Discipline of Zhejiang-A (Zhejiang Gongshang University-Statistics).

\section{References}

1. Asimit, A.V. \& Badescu, A.L. (2010). Extremes on the discounted aggregate claims in a time dependent risk model. Scandinavian Actuarial Journal 2010(2): 93-104.

2. Avram, F., Palmowski, Z., \& Pistorius, M. (2008). A two-dimensional ruin problem on the positive quadrant. Insurance Mathematics \& Economics 42(1): 227-234.

3. Badescu, A.L., Cheung, E.C.K., \& Rabehasaina, L. (2011). A two-dimensional risk model with proportional reinsurance. Journal of Applied Probability 48(3): 749-765.

4. Bingham, N.H., Goldie, C.M., \& Teugels, J.L. (1987). Regular Variation. Cambridge: Cambridge University Press.

5. Chan, W., Yang, H., \& Zhang, L. (2003). Some results on ruin probabilities in a two-dimensional risk model. Insurance Mathematics \& Economics 32(3): 345-358.

6. Chen, Y., Yuen, K.C., \& Ng, K.W. (2011). Asymptotics for the ruin probabilities of a two-dimensional renewal risk model with heavy-tailed claims. Applied Stochastic Models in Business and Industry 27: 290-300.

7. Chen, Y., Wang, L., \& Wang, Y. (2013). Uniform asymptotics for the finite-time ruin probabilities of two kinds of nonstandard bidimensional risk models. Journal of Mathematical Analysis and Applications 401: $114-129$.

8. Chen, Y., Wang, Y., \& Wang, K. (2013). Asymptotic results for ruin probability of a two-dimensional renewal risk model. Stochastic Analysis and Applications 31: 80-91.

9. Embrechts, P., Klüppelberg, C., \& Mikosch, T. (1997). Modelling Extremal Events for Insurance and Finance. Berlin: Springer-Verlag.

10. Foss, S., Dmitry, K., Palmowski, S., \& Rolski, T. (2017). Two-dimensional ruin probability for subexponential claim size. Probability and Mathematical Statistics 37(2): 319-335.

11. Fu, K.A. (2016). On joint ruin probability for a bidimensional Lévy-driven risk model with stochastic returns and heavy-tailed claims. Journal of Mathematical Analysis and Applications 442: 17-30.

12. Gao, Q. \& Yang, X. (2014). Asymptotic ruin probabilities in a generalized bidimensional risk model perturbed by diffusion with constant force of interest. Journal of Mathematical Analysis and Applications 419: $1193-1213$.

13. Hao, X. \& Tang, Q. (2008). A uniform asymptotic estimate for discounted aggregate claims with subexponential tails. Insurance Mathematics \& Economics 43: 116-120.

14. Hu, Z. \& Jiang, B. (2013). On joint ruin probabilities of a two-dimensional risk model with constant interest rate. Journal of Applied Probability 50(2): 309-322.

15. Jiang, T., Wang, Y., Chen, Y., \& Xu, H. (2015). Uniform asymptotic estimate for finite-time ruin probabilities of a time-dependent bidimensional renewal model. Insurance Mathematics ES Economics 64: 45-53.

16. Li, J., Tang, Q., \& Wu, R. (2010). Subexponential tails of discounted aggregate claims in a timedependent renewal risk model. Advances in Applied Probability 42(4): 1126-1146.

17. Tang, Q. \& Tsitsiashvili, G. (2003). Precise estimates for the ruin probability in finite horizon in a discrete-time model with heavy-tailed insurance and financial risks. Stochastic Processes and their Applications 108(2): 299-325.

18. Tang, Q. \& Yuan, Z. (2014). Randomly weighted sums of subexponential random variables with application to capital allocation. Extremes 17(3): 467-493.

19. Zhang, Y. \& Wang, W. (2013). Ruin probabilities of a bidimensional risk model with constant interest rate. Journal of East China Normal University. Natural Science Edition 2013(6): 22-31. 